\title{
Age-of-acquisition effects in reading aloud: Tests of cumulative frequency and frequency trajectory
}

\author{
JASON D. ZEVIN \\ Sackler Institute for Developmental Psychobiology, New York, New York \\ and \\ MARK S. SEIDENBERG \\ University of Wisconsin, Madison, Wisconsin
}

\begin{abstract}
Several studies have reported that the age at which a word is learned affects skilled reading. This ageof-acquisition effect is potentially important for theories of reading and learning. The effect has been difficult to pin down, however, because the age at which a word is learned is correlated with many other lexical properties. Zevin and Seidenberg (2002) analyzed these phenomena, using connectionist models that distinguished between cumulative frequency (the total number of times a word is experienced) and frequency trajectory (the distribution of these experiences over time). The models prompted a reevaluation of the empirical literature on this topic. The present research tested and confirmed three behavioral predictions derived from these models. First, cumulative frequency has an impact on skilled word naming, more so than standard measures of frequency derived from such norms as those of Kučera and Francis (1967). Second, frequency trajectory affects age of acquisition: The timing of exposure to words affects how rapidly they are learned. However, frequency trajectory does not affect skilled reading aloud, because the consistencies in mapping between spelling and sound eventually wash out the effects of early differences in frequency of exposure. Thus, in skilled performance, the timing of exposure to words is less important than the amount of exposure. The results clarify the conditions under which age-dependent learning effects occur in reading aloud.
\end{abstract}

The possibility that some aspects of language learning may be age dependent, in that they can be fully mastered only during a limited window of opportunity in early development, has been widely discussed (e.g., Doupe \& Kuhl, 1999; Flege, Yeni-Komshian, \& Liu, 1999; Johnson \& Newport, 1989; Mayberry, Lock, \& Kazmi, 2002). The hypothesis that there are critical or sensitive periods in language learning has been investigated mainly with regard to phonology, morphology, and syntax; however, lexical learning also needs to be considered. Although the ability to learn new words remains intact well into adulthood (e.g., McCandliss, Posner, \& Givón, 1997; Service \& Craik, 1993), there is evidence that adults process words that were learned early in life more quickly and accurately than words that were learned later (e.g., Brown \& Watson, 1987; Morrison \& Ellis, 1995). This age-of-acquisition (AoA) effect suggests that lexical learning may also be at least partially age dependent: People can continue to learn new words, but there is a lasting advantage for words learned earlier. Converging results from computational modeling and behavioral studies of AoA effects

This research was supported by NIMH Grant P50-MH 64445, NICHD Grant RO1-MH 29891, and an NIMH research scientist development award to M.S.S. Address correspondence to J. D. Zevin, Sackler Institute for Developmental Psychobiology, Weill Medical College of Cornell University, 1300 York Ave., Box 140, New York, NY 10021 (e-mail: jdzevin@lcnl.wisc.edu). suggest new ways of thinking about age-limited learning in word reading and other domains. Connectionist modeling has begun to provide a mechanistic account of changes in the ability to learn over time (Ellis \& Lambon Ralph, 2000; Smith, Cottrell, \& Anderson, 2001) and of the relationship between age-limited learning and generalization (Zevin \& Seidenberg, 2002).

The present study provides behavioral evidence concerning the effects of the frequency and timing of exposure to words on skilled reading aloud. Zevin and Seidenberg's (2002) review of the literature suggested that the behavioral evidence for AoA effects was weak. They pointed out that it is difficult to manipulate AoA while matching stimuli along other dimensions, because AoA is naturally correlated with such variables as imageability, length, and familiarity that also affect skilled performance. These correlations derive from an obvious source: These factors affect the ease of word learning, which is what AoA norms estimate. Thus, it is very difficult to dissociate the effects of when a word was learned (AoA) from the factors that determined when it was learned. Zevin and Seidenberg noted particular difficulties associated with standard measures of word frequency. First, differences among the most commonly used norms with respect to estimates of frequency may result in failures to equate stimuli appropriately with respect to this factor. Second, the theoretical interpretation of such frequency measures is unclear. Do they measure frequency of ex- 
posure to a word in adulthood or since a particular age, the likelihood of recent exposure to a word, or something else? Under what theory of word learning would such factors be relevant?

Zevin and Seidenberg (2002) developed an alternative analysis of these phenomena that relied on two concepts: cumulative frequency and frequency trajectory. Cumulative frequency refers to the total number of exposures to a word. Frequency trajectory refers to the distribution of these exposures over time. Words with the same cumulative frequency may therefore exhibit different trajectories. For example, words such as potty and stroller are very frequent in speech and texts intended for young children but are less frequent in adult discourse; these words are learned relatively early but are used less in adulthood. ${ }^{1}$ Conversely, some words are learned and used more often in adulthood and occur rarely or not at all in childhood (e.g., fax, merlot). In Zevin and Seidenberg's analysis, AoA norms reflect a behavioral outcome, the age at which a word is learned. This outcome is affected by many factors, including frequency trajectory: Other factors aside, words that are experienced more often in childhood will be learned more rapidly. On this view, AoA norms are a surrogate variable for the several aspects of words, including frequency trajectory as well as semantic and phonological factors, that determine when they are learned.

The fact that words have different frequency trajectories creates natural conditions for examining developmental limits on plasticity. Specifically, a residual advantage for words learned early over those learned late would suggest a decrease in the capacity to learn novel words over time. This is similar to the reasoning used in studying whether there are critical periods for learning second languages. There, the assumption is that any influence of AoA on ultimate attainment is due to the developmental state of the organism, not to the difficulty of the language being learned. What one hopes to learn from such studies is how the pattern of exposure to a particular language over the life span influences the ability to achieve native-like proficiency in that language. The aim of manipulating frequency trajectory is much the same: to examine the influence on skilled performance of the developmental state of the individual when specific words are encountered.

Zevin and Seidenberg (2002) conducted a series of simulations using connectionist models that learned mappings from spelling to sound (based on work by Harm $\&$ Seidenberg, 1999). Words trained with a high-to-low frequency trajectory (more frequent early in training) were learned more quickly than items with the complementary trajectory, holding cumulative frequency constant. These manipulations were unaffected by other potentially confounding variables, because a crossed design was used in which each item appeared in each trajectory across runs of the model. Words in the early trajectory were learned more rapidly, but there were no residual effects of trajectory on asymptotic ("adult") performance. In contrast, differences in cumulative frequency of exposure to the same items had a large effect on performance throughout training. Zevin and Seidenberg hypothesized that the lack of a frequency trajectory effect was due to the systematicity of the mapping between spelling and sound: Because this mapping is quasiregular (Seidenberg \& McClelland, 1989), knowledge of the items learned early is helpful in the learning and processing of later items. In a further simulation, the overlap between early and late items was eliminated by artificially manipulating the training set. In this case, frequency trajectory effects were observed, further suggesting that generalization from early to late items was responsible for the lack of frequency trajectory effects in the larger, more realistic models.

Zevin and Seidenberg's (2002) results were somewhat surprising, given that other connectionist models have shown an advantage for patterns learned early in a range of tasks (Ellis \& Lambon Ralph, 2000; Smith et al., 2001). However, these earlier models learned mappings between sets of random bit patterns, which provide no basis for generalization from items learned early to those learned late. Similarly, Monaghan and Ellis (2002) manipulated the consistency of the mappings used in their simulations and found age-limited learning effects for inconsistent mappings only. However, the way in which they manipulated consistency was not directly analogous to the way such effects arise in the mapping from spelling to sound. They used artificial stimuli in which the "vowels" in the inconsistent items could take any of 10 possible pronunciations and each pronunciation was represented by a different, random pattern of activation. This means that among inconsistent items, there was no basis for generalization from the rest of the training set-just the set of circumstances under which Zevin and Seidenberg (2002) predicted that age-limited learning effects should arise.

In the present research, we examined three behavioral predictions derived from Zevin and Seidenberg's (2002) modeling - specifically, (1) frequency trajectory should have an influence on the age at which words are learned, so that words that are experienced more frequently early in life should be learned more rapidly; (2) frequency trajectory should have no residual effect on adult performance in the task of naming aloud, because the advantage for words learned early is washed out by knowledge of the consistencies in the mapping from spelling to sound; and (3) cumulative frequency should have a significant impact on skilled performance.

\section{EXPERIMENT 1}

Zevin and Seidenberg (2002) noted that AoA is essentially an outcome variable. The age at which words are acquired depends on a number of factors, many of which also influence adult processing, such as frequency (McRae, Jared, \& Seidenberg, 1990; Monaghan \& Ellis, 2002; Seidenberg, Waters, Barnes, \& Tanenhaus, 1984), imageability and/or concreteness (Cortese, Simpson, \& 
Woolsey, 1997; Strain, Patterson, \& Seidenberg, 2002; Zevin \& Balota, 2000), length (Spieler \& Balota, 1997), and neighborhood size (Andrews, 1992). Zevin and Seidenberg estimated that approximately $70 \%$ of the variance in AoA can be explained by these standard predictors of adult reading performance.

In Experiment 1, we examined whether frequency trajectory-that is, differences in how exposures to words are distributed over time-has any additional influence on the age at which words are learned. We generated an empirically derived measure of frequency trajectory, using Zeno's (1995) norms. These norms consist of estimates of the frequencies of words at each of 13 grade levels, derived from a corpus of 17 million words obtained from a large sample of texts. These norms (which are similar to the grade-level norms of Carroll, Davies, \& Richman, 1971, but are based on a much larger sample) provide a good approximation of changes in the frequencies of words over time. We also collected new ratings of AoA, imageability, and concreteness for all the words in the study. We then used these measures to predict AoA, with a focus on whether frequency trajectory has an impact independent of the other factors. ${ }^{2}$

\section{Method}

Stimuli. From the Zeno (1995) frequency norms, 328 words that had either low-to-high or high-to-low frequency trajectories were chosen. An item was counted as having a high-to-low frequency trajectory if its frequency in the early grades (1st, 2nd, and 3rd) was three times as great as its frequency in the later grades (11th, 12th, and university level). Low-to-high frequency was defined as the converse pattern of exposure over time. A continuous measure of frequency trajectory was generated for use in regression analyses by subtracting the $\log$ frequency of the word in the latest grades from its $\log$ frequency in the earliest grades.

Subjects. Forty University of Wisconsin, Madison undergraduates participated in each of the three norming studies (120 subjects in all) for course credit. None provided norms for more than one factor.

Procedure. The subjects were instructed to rate items on a scale of 1-7 for one of three factors: imageability, concreteness, or AoA. In each case, the instructions included examples of anchor points on the scales. For imageability, the examples were badger (the school mascot) for high imageability and confusion for low imageability. For concreteness, the examples were cotton and elm for high concreteness and idea and color for low concreteness. For AoA, the early examples were BALL and DOGGIE; late examples were PERPLEX and COGNITIVE. Responses were collected on an iMac running PsyScope 1.5.

\section{Results}

Norms for all the items are available on the Internet at http://lcnl.wisc.edu/people/jdzevin/zs.appendix.html. Table 1 reports means, ranges, and examples of items at the extremes for each factor.

Zero-order correlations among all the variables are presented in Table 2. The two measures of frequency had the highest correlation with rated AoA, followed by frequency trajectory. The contribution of each variable to the predicting of AoA was assessed by examining the change in $R^{2}$ in a series of regression analyses with AoA as the dependent variable and frequency trajectory, cumulative frequency, subjective frequency, length, Coltheart's $N$, imageability, concreteness, and friends-to-neighbors ratio as predictors. The $R^{2}$ value was .797 for the regression of the predictor variables against AoA. Removing frequency trajectory from the analysis resulted in an $R^{2}$ of .703. Thus, the proportion of variance explained by frequency trajectory, with other predictors partialed out, was estimated at $.094[t(319)=12.15, p<.001]$.

Other variables that explained significant proportions of the variance in AoA were cumulative frequency $\left(r^{2}=\right.$ $.018 ; t=5.30, p<.001)$, subjective frequency $\left(r^{2}=.062\right.$; $t=9.89, p<.001)$, imageability $\left(r^{2}=.019 ; t=5.44\right.$, $p<.001)$, and concreteness $\left(r^{2}=.004 ; t=2.67, p<\right.$ $.01)$. Coltheart's $N$ did not predict a significant proportion of the variance $(t=1.53, p>.1)$. Estimates for imageability and concreteness may have been underestimated because they were nearly nonorthogonal $(r=.93$ for the correlation between them). Using an average of the two instead gives an estimate of .036 of the variance explained $(t=7.38, p<.001)$.

The second column in Table 2 shows correlations between frequency trajectory and each of the other predictors. We ran a second set of regression analyses with frequency trajectory as the dependent variable and the remaining five factors (excluding AoA) as predictors. The $R^{2}$ value with all five predictors was .251. Interestingly, significant proportions of the variance in frequency trajectory were explained by imageability $\left[r^{2}=\right.$ $.087 ; t(320)=6.16, p<.001]$, concreteness $\left(r^{2}=.043\right.$; $t=4.28, p<.001)$, length $\left(r^{2}=.023 ; t=3.13, p<\right.$ $.005)$, and $N\left(r^{2}=.010 ; t=2.04, p<.05\right)$. No unique variance was accounted for by the ratio of friends to enemies. Importantly, no unique variance was explained by subjective frequency or cumulative frequency (both $t$ s $<$ 1). Thus, frequency trajectory shares some related factors in common with AoA: Short, imageable, and concrete words tend to be used more frequently in speech and text intended for children. However, the much weaker correlation with measures of cumulative frequency results in a smaller overall $R^{2}$, making frequency trajectory easier to unconfound from related factors than AoA.

The preceding analyses suggest that frequency trajectory influences the age at which words are rated to have been acquired and, because it is only weakly correlated with cumulative frequency and subjective frequency, is less susceptible to confounds with these variables than AoA. We conducted further analyses, using naming latency from two existing studies as dependent variables,

Table 1

Descriptive Statistics for Norms Collected in Experiment 1

\begin{tabular}{lcccc}
\hline & & & \multicolumn{2}{c}{ Examples } \\
\cline { 4 - 5 } \multicolumn{1}{c}{ Factor } & Range & Mean & Low & High \\
\hline AoA & $1.03-6.60$ & 3.51 & boo & ebb \\
Imageability & $1.15-6.84$ & 4.09 & nor & gun \\
Concreteness & $2.00-6.84$ & 4.46 & doubt & eel \\
\hline
\end{tabular}

Note-AoA, age of acquisition. 
Table 2

Correlations Among Norms Collected in Experiment 1 With Other Lexical Variables

\begin{tabular}{lrrrrrrrr}
\hline \multicolumn{1}{c}{ Variable } & \multicolumn{1}{c}{ AoA } & Freq. Traj. & Cum. Freq. & Subj. Freq. & Length & $N$ & Imageability & Concreteness \\
\hline Freq. Traj. & -.5362 & & & & & & & \\
Cum. Freq. & -.6871 & .1054 & & & & & & \\
Subj. Freq. & -.7115 & .1005 & .8354 & & & & & \\
Length & .2869 & -.3196 & -.1342 & -.1358 & & & & \\
$N$ & -.3108 & .3013 & .1791 & .1618 & -.6606 & & & \\
Imageability & -.3048 & .3140 & -.0093 & -.0712 & -.0893 & .0755 & & .1210 \\
Concreteness & -.2112 & .2289 & -.0303 & -.1149 & -.1266 & .1125 & .9309 & .1110 \\
Friends/neighbors & -.1348 & .1992 & .0267 & -.0061 & -.2567 & .3286 & & \\
\hline
\end{tabular}

Note-Freq. Traj., frequency trajectory; Cum. Freq., cumulative frequency from Zeno (1995); Subj. Freq., Balota, Pilotti, and Cortese (2001) subjective frequency; $N$, Coltheart's $N$. Friends/neighbors = summed log frequency of friends divided by summed $\log$ frequency of all neighbors defined at the body level - words with no neighbors assigned a value of 0 .

in order to examine whether it has any effect on skilled adult processing. As can be seen in Table 3, although frequency trajectory is correlated with naming latency in both Seidenberg and Waters's (1989) and Spieler and Balota's (1997) studies, when length, $N$, cumulative frequency, subjective frequency, imageability, concreteness, and proportion of friends to neighbors are also included as predictors, it explains no unique variance in either of the data sets.

\section{Discussion}

Although new norms for AoA, concreteness, imageability, and familiarity were used, along with a new set of items, the results closely replicated those presented by Zevin and Seidenberg (2002), in that the standard lexical predictors-cumulative frequency, length, Coltheart's $N$, imageability, concreteness, and familiarity-accounted for about $70 \%$ of the variance in AoA. These results are also consistent with factor analyses conducted by Bates, Burani, D' Amico, and Barca (2001), which showed that AoA loaded on both a frequency factor and a semantic factor. Frequency trajectory accounted for an additional $9.5 \%$ of the variance when these other variables were taken into account. This result suggests that the pattern of exposures to a word over time has an influence on the age at which it is acquired. Unsurprisingly, words that are frequent early in development are learned earlier than words that are relatively low in frequency early in development.

The analyses also demonstrate that frequency trajectory is less strongly correlated with measures of cumulative and subjective frequency than AoA and that, overall, much less of the variance in frequency trajectory is explained by factors that also influence adult performance. This is methodologically important, because it

Table 3

Unique Variance in Naming Response Time Accounted for by Frequency Trajectory in a Regression Analysis With Six Other Variables

\begin{tabular}{lcc}
\hline \multicolumn{1}{c}{ Study } & $r$ & Unique \\
Variance \\
\hline Seidenberg \& Waters (1989) & -.14 & $.0001, t<1$ \\
Spieler \& Balota (1997) & -.20 & $.0011, t<1$ \\
\hline
\end{tabular}

means that frequency trajectory is more easily manipulated independently of these other factors-in particular, cumulative frequency, which has been confounded with AoA in numerous studies of age-limited learning effects in reading. In Experiment 2, we employed a factorial design to provide a more direct test of this effect.

\section{EXPERIMENT 2}

In Experiment 1, we explored the relationship between frequency trajectory and AoA. The fact that frequency trajectory explains a significant proportion of unique variance in AoA validates our measure of frequency trajectory. The results of Experiment 1 also suggest, albeit preliminarily, that there is no influence of frequency trajectory on adult reading performance. This is consistent with the analyses and modeling work presented by Zevin and Seidenberg (2002). Those models made the further prediction that although frequency trajectory has no influence on adult performance, cumulative frequency does.

Cumulative frequency effects are robust in models trained with a gradient descent learning algorithm, because every exposure to a word improves performance on that particular item, following a power law similar to the power law of practice. Frequency trajectory effects are subtler and arise when the network structure that forms as a result of early learning prevents optimal acquisition of later knowledge. Under some conditions, items trained early may become entrenched, in the sense of interfering with the learning of later items (e.g., Ellis \& Lambon Ralph, 2000). However, these entrenchment effects are highly task and stimulus dependent: They depend on the nature of the mapping between input and output codes. Specifically, according to Zevin and Seidenberg (2002; see also Lambon Ralph \& Ehsan, in press), they are larger for arbitrary mappings (e.g., between objects and their names) than for nonarbitrary mappings (as in the correspondences between spelling and sound in English). In this case, early and later learned do overlap in structure. Thus, what is learned from early words facilitates acquisition of later words and makes generalization (correct performance on novel items) possible. This analysis (Zevin \& Seidenberg, 2002) predicts 
that, whereas frequency affects how rapidly words are learned, it should not affect reading aloud.

Experiment 2 tested the predictions of the models by manipulating cumulative frequency and frequency trajectory independently in a factorial design. Rather than control for AoA, we allowed it to covary with both frequency trajectory and cumulative frequency. On the basis of Experiment 1, we know that both of the latter factors affect AoA. However, our prediction is that only cumulative frequency will have an influence on adult performance. If this pattern of results is observed, it cannot be due to AoA (i.e., the effect of cumulative frequency cannot be a disguised AoA effect), because the AoA disparity is bigger for the frequency trajectory manipulation than for the cumulative frequency manipulation. Hence, the AoA confound would go against the predicted outcome. Moreover, there is additional evidence that cumulative frequency effects can be found with various measures of AoA controlled (e.g., Gerhand \&
Barry, 1998). On the other hand, an effect of frequency trajectory with cumulative frequency controlled would be strong evidence for age-limited learning effects in the reading system.

\section{Method}

Stimuli and Design. One hundred twelve words were selected in a 2 (frequency trajectory) $\times 2$ (cumulative frequency) design. The frequency trajectories are shown separately for high and low cumulative frequency conditions in Figure 1. As is shown in Table 4, stimuli were matched listwise for length and $N$, as well as for onset phoneme and ratio of friends to neighbors. Stimuli are listed by condition on the Internet at http://lcnl.wisc.edu/people/jdzevin/ zs.appendix.html.

Subjective frequency was constant across levels of frequency trajectory, but not across levels of cumulative frequency. AoA was not controlled in either case. To understand why this was not done, consider the graphs in Figure 1. The late-acquired, high-frequency items are more frequent at every grade level than the early-acquired low-frequency items. However, because frequency trajectory is manipulated at each level of cumulative frequency, a main effect of
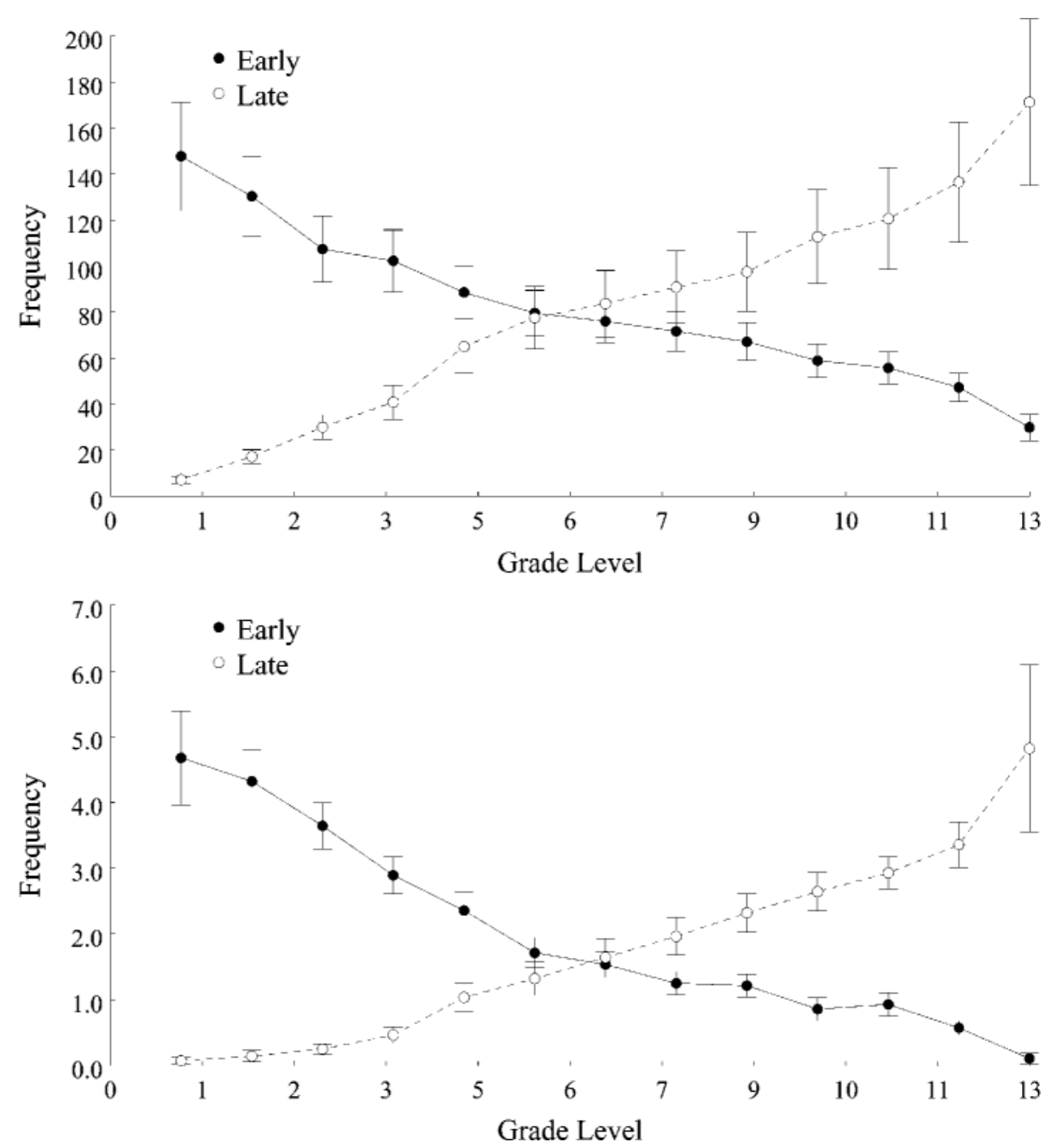

Figure 1. Frequency trajectories of items in Experiment 2. Top: high-frequency items. Bottom: low-frequency items. Error bars represent standard errors. 
Table 4

Descriptive Statistics for Items in Experiment 2

\begin{tabular}{lllllll}
\hline \multicolumn{1}{c}{ Factor } & HF & LF & Difference & Early & Late & Difference \\
\hline Cumulative frequency & 6.68 & 3.13 & $3.35^{* *}$ & 4.98 & 4.83 & 0.15 \\
Subjective frequency & 4.77 & 3.43 & $1.34^{* *}$ & 4.04 & 4.15 & -0.11 \\
AoA & 3.40 & 4.35 & $-0.95^{* *}$ & 3.29 & 4.67 & $-1.38^{* *}$ \\
Length & 4.4 & 4.4 & 0 & 4.3 & 4.5 & -0.2 \\
$N$ & 5.6 & 5.2 & 0.4 & 5.7 & 5.0 & 0.7 \\
Imageability & 3.97 & 4.06 & -0.09 & 4.48 & 3.55 & $0.93^{* *}$ \\
Concreteness & 4.37 & 4.48 & -0.11 & 4.67 & 4.18 & $0.49^{*}$ \\
Friends/neighbors & 0.76 & 0.86 & -0.10 & 0.83 & 0.79 & 0.04 \\
\hline
\end{tabular}

Note-Cumulative frequency $=\log ($ Zeno, 1995) frequency, summed across all grades; subjective frequency estimates are from Balota, Pilotti, and Cortese (2001). Figures for cumulative frequency are collapsed across frequency trajectory manipulations and vice versa in order to highlight manipulations for main effects. AoA, age of acquisition; HF, high frequency; LF, low frequency. $* p<.05 . \quad * * p<.001$.

frequency trajectory would still indicate an effect of age-limited learning.

Finally, in order to have a large enough list of stimuli, concreteness and imageability were allowed to covary with frequency trajectory, so that items acquired early were both more imageable and more concrete than items acquired late. Because semantic variables have only weak effects on reading aloud, particularly for consistent words (Strain, Patterson, \& Seidenberg, 1995; Zevin \& Balota, 2000), it was preferable to match the lists carefully for length, $N$, and cumulative frequency (where appropriate) and to control for concreteness and imageability statistically. This was accomplished by running an analysis of covariance (ANCOVA) including concreteness and imageability as continuous variables. All $F$ s are, therefore, based on items analyses in which discrete factors have been assigned dummy variables and semantic factors included as continuous measures.

Subjects. Thirty-eight University of Wisconsin, Madison undergraduates participated in the naming study for course credit or a $\$ 5$ remuneration.

Procedure. Words were presented using PsyScope 1.5 on an iMac computer. Word lists were randomized anew for each subject. The subjects were seated a comfortable distance from the computer and were instructed to read each word aloud as quickly and accurately as possible. On each trial, a fixation cross was presented for $500 \mathrm{msec}$, followed by a word. Eight practice trials were run to allow the subjects to become accustomed to the procedure. Response times were recorded using the PsyScope button box and were scored for accuracy on line by the experimenter. Sessions were taped to allow off line revisions of these scores.

\section{Results}

As is shown in Figure 2, a robust (16-msec) effect of cumulative frequency was present, whereas no effect of frequency trajectory was present. Only the main effect of cumulative frequency was significant in the ANCOVA on response latencies $[F(1,106)=6.22, p<.05]$. Neither the main effect of frequency trajectory nor the interaction between frequency trajectory and cumulative frequency was reliable (both $F \mathrm{~s}<1$ ). Effects of the covariates (imageability and concreteness) were also nonsignificant (both $F \mathrm{~s}<1$ ). The mean proportion of errors was $2 \%$ in each condition. There were no effects of any kind in the error data.

\section{Discussion}

The results of Experiment 2 were clear. There was a strong effect of cumulative frequency, in accord with

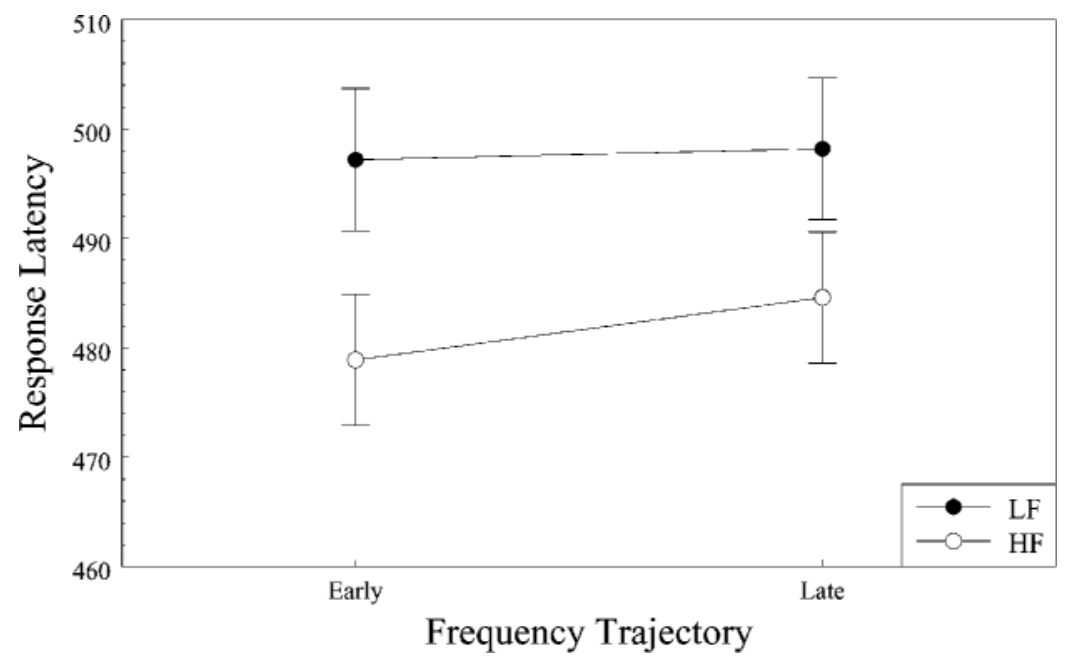

Figure 2. Adjusted means for Experiment 2. HF, high (cumulative) frequency; LF, low (cumulative) frequency. 
models in which repetitions of stimuli improve performance. Unlike statistics such as those of Kučera and Francis (1967), the cumulative frequency measure reflects exposures over a longer time span and, thus, suggests that frequency effects extend further in time than is usually assumed. In contrast, although Experiment 1 indicated that frequency trajectory is predictive of AoA, we found no effect of frequency trajectory on skilled performance. This corroborates the regression analyses presented above, as well as the analyses and simulation results of Zevin and Seidenberg (2002).

Interpreting null results can be difficult, because inferential statistics that test their reliability are less developed than those for positive results. One way to determine the reliability of a null result is to conduct a comparison of effect sizes across a range of studies of a similar kind. Table 5 displays effect sizes for a number of studies that have examined cumulative frequency and AoA, along with the present study. The last column shows the frequency discrepancy for each set of items. The table shows that the effect sizes for both AoA and frequency tracked the size of the manipulation of cumulative frequency, with large effect sizes occurring with large differences in cumulative frequency. In contrast, the effect size for our frequency trajectory manipulation and the consistent items from Monaghan and Ellis are much smaller than the other effect sizes. These are also the only manipulations that did not result in a large disparity between early and late items in cumulative frequency (Zevin \& Seidenberg, 2002).

\section{GENERAL DISCUSSION}

The present results corroborate a number of the predictions and assumptions of the modeling work presented in Zevin and Seidenberg (2002). In Experiment 1, a measure of frequency trajectory was developed that explains a substantial proportion of unique variance in the age at which words are acquired, even when other measures that explain $70 \%$ of the variance in AoA are partialed out. This suggests that our measure of frequency trajec-

Table 5

Effect Size (on Response Time) and the Disparity in Cumulative Frequency in Studies of Age-Limited Learning Effects in Reading Aloud

\begin{tabular}{llcc}
\hline \multicolumn{1}{c}{ Study } & Factor & $\begin{array}{c}\text { Effect } \\
\text { Size }(f)\end{array}$ & $\begin{array}{c}\text { Frequency } \\
\text { Disparity }\end{array}$ \\
\hline Gerhand \& Barry (1998) & Frequency & .35 & 2.90 \\
& AoA & .21 & 1.80 \\
Monaghan \& Ellis (2002) & Frequency & .20 & 2.39 \\
Inconsistent items & AoA & .20 & 1.84 \\
Consistent items & AoA & .07 & 0.46 \\
Present results & Frequency & .23 & 3.35 \\
& Frequency & & \\
& trajectory & .04 & 0.15 \\
\hline
\end{tabular}

Note: $f=$ effect size; frequency disparity $=\log$ of Zeno (1995) frequency of high-frequency (or early) items less log frequency of low-frequency (or late) items. AoA, age of acquisition. tory is valid, insofar as the pattern of exposure to words over the life span should have a strong influence on when words are acquired. In Experiment 2, frequency trajectory was manipulated orthogonally to cumulative frequency. Whereas a large cumulative frequency effect was found, no effect of frequency trajectory was found. Thus, the pattern of exposure to words over time influences AoA, but not skilled performance.

These results contrast with a number of studies in which independent effects of AoA and frequency have been found (Gerhand \& Barry, 1998; Morrison \& Ellis, 1995). Paradoxically, by using rated norms (or objectively determined norms) for AoA, those studies did not provide the strongest test of the AoA hypothesis. Because AoA itself is so strongly correlated with other factors that also predict adult performance, it is extremely difficult to perform experiments in which AoA is manipulated while other factors are held constant. As a result, all but one of the previous studies had confounded AoA and frequency. Interestingly, when cumulative frequency was appropriately controlled in a study by Monaghan and Ellis (2002), no AoA effect was found. Because frequency trajectory is less strongly correlated with factors that influence adult performance, it allows a more stringent test of the hypothesis that age-limited learning plays a role in lexical processing.

Although it is difficult to argue conclusively for the null hypothesis, three aspects of the present data force us to conclude that frequency trajectory has no influence on adult reading performance. First, we found no evidence of frequency trajectory effects in two different experiments, one of which was replicated using two different data sets. Second, the factorial design of Experiment 2 provided enough statistical power to detect effects of cumulative frequency, which are typically quite weak for consistent words. The null effect of frequency trajectory in this experiment is, thus, unlikely to be the result of a Type II error. At the very least, we have shown the effect of frequency trajectory to be much weaker than the effect of cumulative frequency - in contrast to previous reports in which effects of AoA were typically equal to or larger in size than frequency effects. Finally, the results of the experiments support predictions derived from a model that covers a broad range of other phenomena (Harm, 1998; Harm \& Seidenberg, 1999). The effect of frequency trajectory on AoA, the main effect of cumulative frequency, and the absence of a frequency trajectory effect are all explicit predictions of the modeling work reported by Zevin and Seidenberg (2002).

Because frequency trajectory is a strong predictor of AoA and yet is less strongly correlated with factors that influence both AoA and adult processing, it provides a unique tool for examining age-limited learning effects. Experiments in which the methodology introduced here is used can help advance the understanding of the mechanisms underlying age-limited learning more generally by determining which tasks do (and do not) give rise to frequency trajectory effects. 


\section{REFERENCES}

ANDREWS, S. (1992). Frequency and neighborhood effects on lexical access: Lexical similarity or orthographic redundancy. Journal of Experimental Psychology: Learning, Memory, \& Cognition, 18, 234-254.

Balota, D., Pilotti, M., \& Cortese, M. J. (2001). Subjective frequency estimates for 2,938 monosyllabic words. Memory \& Cognition, 29, 639-647.

Bates, E., Burani, C., D' Amico, S., \& Barca, L. (2001). Word reading and picture naming in Italian. Memory \& Cognition, 29, 986-999.

BROWN, G. D. A., \& WATSON, F. L. (1987). First in, first out: Word learning age and spoken word-frequency as predictors of word familiarity and word naming latency. Memory \& Cognition, 15, 208-216.

Carroll, J. B., Davies, P., \& Richman, B. (1971). The American Heritage word frequency book. Boston: Houghton-Mifflin.

Cortese, M. J., Simpson, G. B., \& Woolsey, S. (1997). Effects of association and imageability on phonological mapping. Psychonomic Bulletin \& Review, 4, 226-231.

Doupe, A. J., \& KuHL, P. K. (1999). Birdsong and human speech: Common themes and mechanisms. Annual Review of Neuroscience, 22, 567-631.

ELLIS, A. W., \& LAMBON RALPH, M. A. (2000). Age of acquisition effects in adult lexical processing reflect loss of plasticity in maturing systems: Insights from connectionist networks. Journal of Experimental Psychology: Learning, Memory, \& Cognition, 26, 1103-1123.

Flege, J. E., Yeni-Komshian, G. H., \& LiU, S. (1999). Age constraints on second-language acquisition. Journal of Memory \& Language, 41, 78-104.

GERHAND, S., \& BARRY, C. (1998). Word frequency effects in oral reading are not merely age-of-acquisition effects in disguise. Journal of Experimental Psychology: Learning, Memory, \& Cognition, 24, 267283.

HARM, M. W. (1998). Division of labor in a computational model of visual word recognition. Unpublished doctoral dissertation, University of Southern California.

Harm, M. W., \& Seidenberg, M. S. (1999). Phonology, reading, and dyslexia: Insights from connectionist models. Psychological Review, 163, 491-528.

JOHNSON, J. S., \& NEWPORT, E. L. (1989). Critical period effects in second language learning: The influence of maturational state on the acquisition of English as a second language. Cognitive Psychology, 21, 60-99.

KUČERA,H., \& FrANCIS, W. N. (1967). Computationalanalysis of presentday American English. Providence, RI: Brown University Press.

LAMBON RALPH, M. A., \& EHSAN, S. (in press). Age of acquisition effects depend on the mapping between representations and the frequency of occurrence: Empirical and computational evidence. Visual Cognition.

MAYBERRY, R. I., LOCK, E., \& KAZMI, H. (2002). Development: Linguistic ability and early language exposure. Nature, 417, 38 .

MCCANDliss, B. D., Posner, M. I., \& Givón, T. (1997). Brain plasticity in learning visual words. Cognitive Psychology, 33, 88-110.

MCRAe, K., JARED, D., \& SeIdenberG, M. S. (1990). On the roles of frequency and lexical access in word naming. Journal of Memory \& Language, 29, 43-65.

Monaghan, J., \& Ellis, A. W. (2002). What exactly interacts with spelling-sound consistency in word naming? Journal of Experimental Psychology: Learning, Memory, \& Cognition, 28, 183-206.

MORRISON, C. M., \& ElLis, A. W. (1995). Roles of word frequency and age of acquisition in word naming and lexical decision. Journal of Experimental Psychology: Learning, Memory, \& Cognition, 21, 116153.

MORRISON, C. M., \& ELLIS, A. W. (2000). Real age of acquisition effects in word naming and lexical decision. British Journal of Psychology, 91, 167-180.
Morrison, C. M., Ellis, A. W., \& Chappell, T. D. (1997). Age of acquisition norms for a large set of object names and their relation to adult estimates and other variables. Quarterly Journal of Experimental Psychology, 50A, 528-559.

SEIDENBERG, M. S., \& MCClelland, J. L. (1989). A distributed, developmental model of word recognition and naming. Psychological Review, 96, 523-568.

SeidenberG, M. S., \& Waters, G. S. (1989). Reading words aloud: A mega study [Abstract]. Bulletin of the Psychonomic Society, 27, 489.

Seidenberg, M. S., Waters, G. S., Barnes, M. A., \& Tanenhaus, M. K. (1984). When does irregular spelling or pronunciation influence word recognition? Journal of Verbal Learning \& Verbal Behavior, 23, 383-404.

Service, E., \& Craik, F. I. M. (1993). Differences between young and older adults in learning a foreign vocabulary. Journal of Memory \& Language, 32, 608-623.

Smith, M. A., Cottrell, G. W., \& Anderson, K. L. (2001). The early word catches the weights. In T. K. Leen, T. G. Dietterich, \& V. Tresp (Eds.), Advances in neural information processing systems 13 (pp. 5258). Cambridge, MA: MIT Press.

SPIELER, D. H., \& BALOTA, D. A. (1997). Connectionist models of word naming: An examination of item level performance. Psychological Science, 8, 411-416.

Strain, E., Patterson, K., \& Seidenberg, M. S. (1995). Semantic effects in single-word naming. Journal of Experimental Psychology: Learning, Memory, \& Cognition, 21, 1140-1154.

Strain, E., Patterson, K., \& Seidenberg, M. S. (2002). Theories of word naming interact with spelling-sound consistency. Journal of Experimental Psychology: Learning, Memory, \& Cognition, 28, 207-214. ZENO, S. (ED.) (1995). The educator's word frequency guide. Brewster, NJ: Touchstone Applied Science Associates.

ZEVIN, J. D., \& BALOTA, D. A. (2000). Priming and attentional control of lexical and sublexical pathways during naming. Journal of Experimental Psychology: Learning, Memory, \& Cognition, 26, 121-135. ZE VIN, J. D., \& SEIDENBERG, M. S. (2002). Age-of-acquisition effects in reading and other tasks. Journal of Memory \& Language, 47, 1-29.

\section{NOTES}

1. Such words exhibit large but relatively brief increases in frequency among adults who are parents of young children.

2. Some studies have used objective AoA norms based, for example, on the average age at which children could use a word correctly to name an object (Morrison \& Ellis, 2000). Such norms were not available for many of the items that had the critical patterns of frequency trajectory we selected for. A number of studies have shown that rated AoA and objective AoA are very strongly related (Morrison \& Ellis, 2000; Morrison, Ellis, \& Chappell, 1997). Rated AoA measures are potentially problematic because they involve collecting estimates from the same population that provides estimates of other variables (notably, imageability and concreteness); it may be that these AoA norms are "contaminated" by other factors in a way that objective norms are not (note, e.g., that correlations with frequency are higher for rated than for objective measures of AoA, whereas correlations with imageability are higher in the objective norms; Morrison et al., 1997). However, our argument is that the main problem with AoA is its status as an outcome measure- that is, that it is the result of other factors that make some words easier to learn than others. This is doubly true of objective measures, where the methodology involves using a performance measure from children (the average age of children who can correctly name a picture) to predict a performance measure in adults (response latency and accuracy in word naming).

(Manuscript received February 17, 2003; revision accepted for publication August 28, 2003.) 